\title{
A Quantitative Survey of various Fingerprint Enhancement techniques
}

\author{
Kumud Arora \\ Inderprastha Engg. College \\ Ghaziabad, INDIA
}

\author{
Dr.Poonam Garg \\ IMT \\ Ghaziabad, INDIA
}

\begin{abstract}
Image Preprocessing is an important step in the area of image processing and pattern recognition. This paper aims to present a review of recent as well as classic fingerprint image enhancement techniques. The umbrella of techniques used for evaluation varies from histogram based enhancement, frequency transformation based, Gabor filter based enhancement and its variants to composite enhancement technique. The effectiveness of enhancement techniques proposed by various researchers is evaluated on the basis of peak signal to noise ratio and equal error rate which refers to robustness and stability of identification process. Experimental results shows that incorporating the enhancement technique based on Gabor filter in wavelet domain and composite method improves equal error rate .Improved error rate and peak signal noise ratio improves the identification/verification accuracy marginally. The major goal of the paper is to provide a comprehensive reference source for the researchers involved in enhancement of fingerprint images which is essential preprocessing step in automatic fingerprint identification and verification.
\end{abstract}

\section{General Terms}

Fingerprint Enhancement Techniques

\section{Keywords}

WFT (Windowed Fourier Transformation), Gabor Filters, PSNR( Peak Signal-to-noise ratio),EER(Equal Error Rate)

\section{INTRODUCTION}

Wide usage of biometric information for person identity verification purposes, terrorist act prevention measures and authentication process simplification in computer systems has raised significant attention to reliability and efficiency of biometric systems. Biometric science utilizes the measurements of a person's behavioral characteristics (keyboard strokes, mouse movement) or biological characteristics (fingerprint, iris, nose, eyes, jaw, voice pattern, etc). Fingerprint is one of the most promising methods among biometric techniques and has been used for individual authentication since $19^{\text {th }}$ century. The two fundamental premises on which fingerprint recognition is based are: fingerprint details are permanent and fingerprints of individuals are unique. A fingerprint is formed of a group of curves .Historically, in law enforcement applications the acquisitions of fingerprint images was performed by using inktechnique. Now days they are captured as live-scan digital images acquired by directly sensing the fingerprint surface with an electronic fingerprint scanner.The fingerprint pattern displays different features at different levels. At level 1, the most evident structural characteristic of fingerprint is a pattern of the lines (ridges) flowing in various patterns. This level is also known as global level of fingerprint analysis. At this level fingerprint pattern exhibits one or more regions where ridge lines are characterized by high curvature and frequent termination. These regions or singular regions are commonly used for assigning a fingerprint to a set of five distinctive classes (arch, tented arch, left loop, right loop, double loop and whorl) with the aim of simplifying search and retrieval. At local level or level 2 fingerprint images are characterized by discontinuity of ridges. The two most prominent ridge characteristic, called minutiae are ridge termination and ridge bifurcation.

A typical fingerprint-based recognition system works in two distinct modes: enrollment and recognition. The purpose of the enrollment mode is to create a database. During this mode, an enrollee fingerprint is captured and processed in three stages: Fingerprint Reading, Image Preprocessing, and Feature Extraction. After the feature extraction stage, a set of representative features of the enrollee fingerprint is saved in the database. During the recognition mode, a fingerprint to be recognized undergoes the same three processing steps as in the enrollment mode. The result is compared with a feature set template from the database in the feature matching stage. A match score which measures the similarity between the query feature set and database feature set is calculated. Higher values indicate higher confidence in a match.

\section{FINGERPRINT ENHANCEMENT}

Although fingerprint recognition is one of the earliest applications of pattern recognition, the accuracy of state-of-theart fingerprint-matching systems is still not comparable to human fingerprint experts in many situations. Distortions are inevitable when mapping a three dimensional fingertip onto a two dimensional plane. These can be caused by varying vertical pressures, shear forces and impression conditions. Their quality is therefore not satisfactory for accurate processing. Their quality is therefore not satisfactory for accurate processing. For this reason image preprocessing is a crucial part of a fingerprintbased recognition system. It aims at reducing noise, enhancing the quality of the captured fingerprint image, and facilitating the subsequent processing steps. Fingerprint enhancement is to improve the clarity of ridge structures of input fingerprint images and to facilitate the extraction of ridges and minutiae from the improved ridge structures of fingerprint images in recoverable regions and to remove the unrecoverable regions .The basic fingerprint image preprocessing operations are: Noise Reduction and contrast enhancement which enhances the contrast between darker and brighter curves in a fingerprint image, darker curves. There appear many algorithms and techniques proposed and applied to fingerprint image Enhancement : using Fourier transform [4, 5], Gabor filters [2, 6], Wavelet transform [7, 8, 9, 10, 11], and minutiae filtering, applied to binary [12] or gray-scale images [13]. 


\section{FINGERPRINT IMAGE TECHNIQUES UNDER EVALUATION}

\subsection{WFT Based Fingerprint Image Enhancement}

A frequency transformation decomposes an image from its spatial-domain form of bright intensities into a frequencydomain form of frequency components [3]. The frequency domain shows the frequency of brightness variations, the direction of variation patterns, and the amplitude of the waveforms representing the patterns. One common motive to generate image frequency spectrum data is to filter the data. The process of filtering in frequency (spectral) domain [9] can be summarized as :

- Transform image data to the frequency domain via the Fast Fourier Transform (FFT).

- Multiply (convolve) the image's spectrum with some filtering mask.

- Transform the filtered spectrum back to the spatial domain.

The FFT filter based fingerprint image enhancement is based upon non stationary directional Fourier domain filtering. Fingerprints are first smoothed using a directional filter whose orientation is everywhere matched to the local ridge orientation. Thresholding then yields the enhanced image. The twodimensional Fourier transform of is defined by the relationship:

$$
G\left(p_{1}, q_{1}\right)=\sum_{p=0}^{M-1} \sum_{q=0}^{N-1} f(p, q) \times \exp \left\{-2 t \Pi \times\left(\frac{p_{1} p}{M}+\frac{q_{1} q}{N}\right)\right\}
$$

for $\mathrm{p} 1=1 \ldots \mathrm{W}, \mathrm{q} 1=1,2 . . \mathrm{W}$, where $\mathrm{W}$ is the block size when image is divided into non overlapping blocks. FFT of fingerprint image is achieved block wise when image is divided into blocks of size 32 or 16.In order to enhance a specific block by its dominant frequencies; we multiply the FFT of the block by its magnitude a set of times. Where

$$
\mathrm{FFT}=\operatorname{abs}\left(\mathrm{G}\left(\mathrm{p}_{1}, \mathrm{q}_{1}\right)\right)=\left|\mathrm{G}\left(\mathrm{p}_{1}, \mathrm{q}_{1}\right)\right|
$$

The block is enhanced according to:

$$
g(p, q)=G^{-1}\left\{G\left(p_{1}, q_{1}\right) \times\left|G\left(p_{1}, q_{1}\right)\right|^{n}\right\}
$$

Where $\mathrm{G}^{-1}(\mathrm{G}(\mathrm{p} 1, \mathrm{q} 1))$ is done by:

$$
f(p, q)=\frac{1}{M N} \sum_{p=0}^{M=1} \sum_{q=0}^{N=1} G(p 1, q 1) \times \exp \left\{t 2 \Pi \times\left(\frac{p_{1} p}{M}+\frac{q_{1} q}{N}\right)\right\}
$$

Frequency domain techniques are less efficient computationally and require more processing resources to implement.

\begin{tabular}{|l|l|l|}
\hline $\begin{array}{l}\text { FFT } \\
\text { Enhanced } \\
\text { Image }\end{array}$ & $\begin{array}{l}\text { FFT spectrum } \\
\text { of Original } \\
\text { Image }\end{array}$ & $\begin{array}{l}\text { FFT spectrum } \\
\text { of enhanced } \\
\text { image }\end{array}$ \\
\hline
\end{tabular}

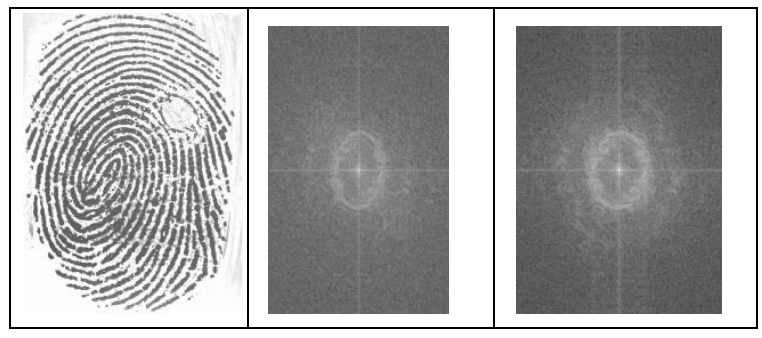

Fig 1: WFT enhanced image and its spectrum

\subsection{GABOR Filters Based Fingerprint Enhancement}

If enhancement stage uses a single filter convolution on the entire fingerprint image, it create significant number of spurious minutiae, a large number of genuine minutiae are missed and large error in the location (position and orientation) of minutiae is introduced. Most of the fingerprint enhancement techniques use contextual filter or multi-resolution filter. Several types of filters in both spatial and frequency domains have been proposed in the literature. The purpose of the filters is to fill small gaps (low-pass effect) in the direction of the ridge and to increase the discrimination (band-pass effect) between ridge and valleys in the direction, orthogonal to the ridge[1]. Hong et. al. [2] introduced Gabor filters for fingerprint enhancement algorithm which decomposes the input fingerprint image into a set of filtered images. Gabor filters [10] have both frequency selective and orientation selective properties and have optimal joint resolution in both spatial and frequency domains. A Gabor filter can be viewed as a sinusoidal plane of particular frequency and orientation, modulated by a Gaussian envelope. It can be written as: $\quad \mathbf{H}(\mathbf{x}, \mathbf{y})=\mathbf{S}(\mathbf{x}, \mathbf{y}) \times \mathbf{G}(\mathbf{x}, \mathbf{y})$, where $S(x, y)$ is a complex sinusoid, known as a carrier, and $\mathrm{G}(\mathrm{x}, \mathrm{y})$ is a 2-D Gaussian shaped function, known as envelope. An even symmetric Gabor filter has the following general form in the spatial domain [2]: $u(x, y, \theta)=x \cos \theta+y \sin \theta, v(x, y, \theta)=-x \sin \theta+y \cos \theta$

$$
h(x, y, \theta, f)=\frac{1\left(\sigma_{u} \sigma_{v}\right)}{2 \pi} * \exp \left\{-\frac{1}{2}\left(\frac{u^{2}}{\sigma_{u}^{2}}+\frac{v^{2}}{\sigma_{v}^{2}}\right)\right\} * \cos (\mathrm{fu}),
$$

where $\theta$ and $f$ are corresponding orientation and frequency, $\sigma_{u}$ and $\sigma_{\mathrm{v}}$ are gaussian envelop constants. The sinusoidal shaped waves of ridges and furrows in fingerprint image vary slowly in a local constant orientation. Therefore, a band-pass filter that is tuned to the corresponding frequency and orientation can efficiently remove the undesired noise and preserve the true ridge and furrow structures. Gabor filters can be used as bandpass filters to remove the noise and preserve true ridge/valley structures. The convolution nature of Gabor filter contributes to high computational complexity which in turn leads to overall increase in running time of identification/verification process.

Another difficulty in application of multi-resolution or Gabor filters lies in feeding reliable estimation inputs of the local context, i.e. the local orientation and ridge frequency to Gabor Filters. Failure to correctly estimate the local context can lead to the creation of artifacts in the enhanced image which consequently tends to increase the number of identification or verification errors. Following are the filtered images at different orientations 0,22.5,45,90 degrees successively and below are original image and combined final enhanced image: 


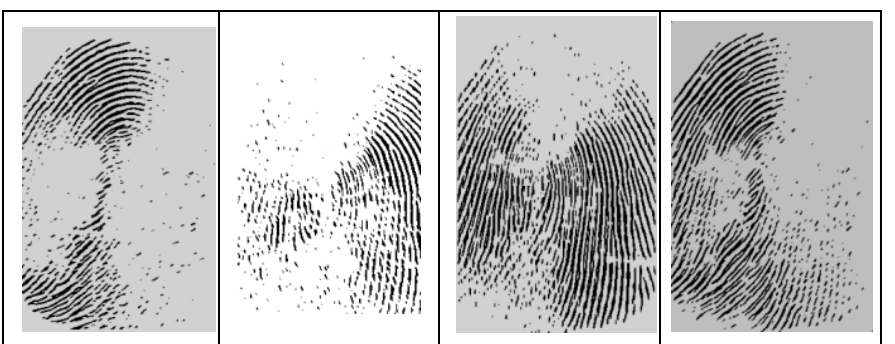

Fig.2 Gabor Filtered Images at various orientations $(0,22.5,45,90$ degrees successively)

\subsection{Fast implementation of Gabor filter}

Zhu etal[14] employed a fast implementation of Gabor filter to reduce the computational complexity. The symmetric property of gabor filters is exploited for fast implementation. Because Gabor filter is even symmetric, the procedure of filtering is implemented only in one quadrant region other than in the whole scope. The other quadrants values are derived from filtered quadrant image.
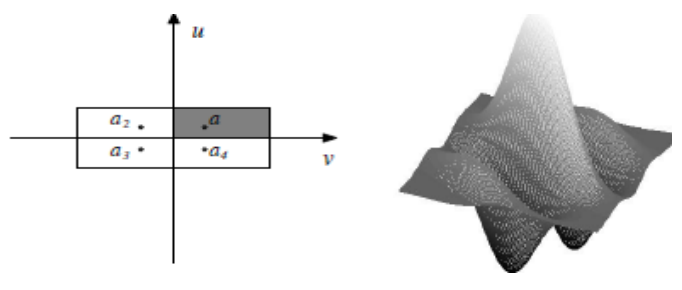

Fig.3 Symmetric Feature of Gabor Filter

After tuning the orientation field and ridge frequency of fingerprint, designed for Gabor filter, the filtering region is regulated according to the orientation and frequency of local ridges instead of traditional fixed region .The filtering region is implemented as a transformable rectangle, where the length is along the orientation of the ridge and the width is along the corresponding perpendicular orientation. Using this method, only $1 / 4$ of the filtering region has to be calculated. Zhu etal[14] reported the average reduction in cost of computation time by $32 \%$.

\subsection{Log-Gabor filter in fingerprint image enhancement}

Gabor filters have two main limitations. The maximum bandwidth of a Gabor filter is limited to approximately one octave and Gabor filters are not optimal if one is seeking broad spectral information with maximal spatial localization. And unfortunately, the signal orthogonal to the local orientation in fingerprint images does not always consist of an ideal digital sinusoidal plane wave (Yang et al.[6]). This means that there is a certain bandwidth of the signal orthogonal to the ridge orientation in the local region of fingerprint images. To overcome the limitations of traditional Gabor filter and promote fingerprint enhancement performance Wang etal [16] introduces the Log-Gabor filter. Log-Gabor filters can be constructed with arbitrary bandwidth which can be optimized to have minimal spatial extent and are allowed to reduce the over-representation of low frequencies.

Log-gabor filters are constructed in the frequency domain. The original fingerprint image is transformed to frequency domain by implementing the windowed Fourier transform (WFT) .The frequency spectrum is filtered with a bank of Log-Gabor filters . The 2D Log-Gabor filter in polar coordinates system, can be divided into two components: the radial filter and the angular filter. The radial filter has a frequency response described by

$$
G_{r(r)}=\exp \left(-\frac{\left(\log \left(\frac{r}{f_{o}}\right)\right)^{2}}{2 \sigma_{r}^{2}}\right)
$$

and the angular filter has a frequency response described by

$$
G_{\theta(\theta)}=\exp \left(-\frac{\left(\theta-\theta_{\theta}\right)^{2}}{2 \sigma_{\theta}^{2}}\right)
$$

The two components are multiplied together to construct the overall Log-Gabor filter which has the transfer function as $G(r, \theta)=G_{r(r)} * G_{\theta(\theta)}$, where $(\mathrm{r}, \quad \theta)$ represents the polar coordinates, $\mathrm{f} 0$ is the center frequency of the filter, $\Theta_{0}$ is the orientation angle of the filter, $\sigma_{\mathrm{r}}$ determines the scale bandwidth and $\sigma_{\theta}$ determines the angular bandwidth. $\mathrm{f} 0$ and $\Theta_{0}$ respectively correspond to the local frequency and orientation of fingerprint images, $\sigma_{\mathrm{r}}$ and $\sigma_{\Theta}$ are specified according to the empirical data for fingerprint images. The scale bandwidth of the Log-Gabor filter is set by specifying the parameter $\sigma_{\mathrm{r}}$ which is the standard deviation of the radial filter. The larger the $\sigma_{\mathrm{r}}$ value is, the larger the scale bandwidth will be. Empirically, a $\sigma_{\mathrm{r}}$ value of 0.3 will result in a filter with a bandwidth of approximately one octave and a value of 0.6 will result a bandwidth of roughly two octaves. From the filtering results of fingerprint images, we have seen that larger $\sigma_{\mathrm{r}}$ value helps to preserve the ridge structure but simultaneously impairs the denoise performance. To provide a suitable trade-off, the value of $\sigma_{\mathrm{r}}$ was set to 0.5 [6]. The angular bandwidth of the Log-Gabor filter is controlled by the parameter $\sigma_{\theta}$ which is the standard deviation of the angular filter. The larger $\sigma_{\theta}$ is the larger the angular bandwidth. Based on the empirical data, the value of $\sigma_{\mathrm{e}}$ was set to pi /9 in low curvature region and was increased to $\mathrm{pi} / 5$ in high curvature region. Let $\mathrm{F}(\mathrm{u}, \mathrm{v})$ and $\mathrm{F}_{0}(\mathrm{u}, \mathrm{v})$ respectively denote the original spectrum and filtered spectrum, the filtering can be expressed as

$F_{0}(u, v)=G(u, v) \cdot F(u, v)$,

where $\mathrm{G}(\mathrm{u}, \mathrm{v})$ is the expression of the Log-Gabor filter in Cartesian coordinates system. Following is the enhanced image obtained by using Log Gabor filters

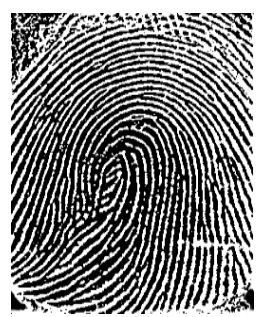

Fig.4 Enhanced Image by Log-Gabor filters 


\subsection{Gabor Filter Based Enhancement in Wavelet Domain}

In this approach a normalized fingerprint image is decomposed into approximation and detail sub-images. The wavelet transform is implemented by a pyramidal algorithm, which is based on convolution with quadrature mirror filters (QMFs). The choice of wavelet base filter defines the information of detail sub-images. The wavelet base filter can be chosen from 294 wavelet different families .In approach used by [15] db7 wavelet is chosen. After decomposition the orientation is estimated for the approximation image. The fingerprint approximation sub-image contains fewer noises, so the orientation estimated from approximation is more reliable. Finally, Gabor filter is used to enhance the fingerprint image in wavelet domain and the fingerprint is reconstructed. The enhanced images in wavelet domain use wavelet reconstruction to get the final enhanced fingerprint image. Algorithm's time cost is reduced by $30 \%$ as orientation is estimated from the approximation sub-image, which is a quarter of original fingerprint image.
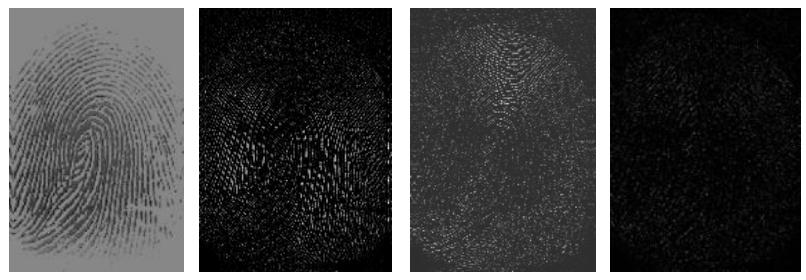

Fig.5 Decomposed Fingerprint Image by $\mathrm{Db} 7$

\subsection{Fingerprint Image Enhancement using Composite Method}

Kale etal[16] used a composite method based on the contrast enhancement, frequency and spatial domain filtering, and quick mask on gray scale images .For intensity mapping the intensity stretching technique with gamma operator is used.The frequency spectrum obtained by fourier transformation of intensity stretched fingerprint image. The spectrum is convolved with high pass filter function for increasing sharpness of image. Inverse of fourier spectrum of image is obtained by applying inverse fourier transformation. The inverse FFT image is convolved with quick mask edge detector which detects edges in all eight directions in one convolution. Average filtering is used for smoothing the ridges .The method used in [16] shows better performance for enhancement for both the fingerprint images obtained from oily, dry or wet fingers and also connects the broken ridges in noisy images.

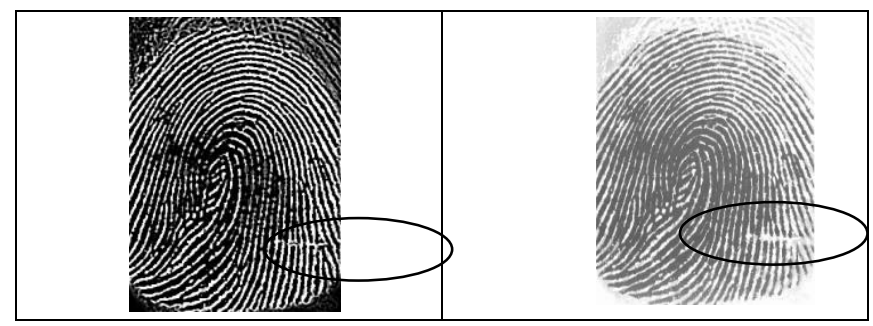

Fig.6 (a) and (b) Enhanced Image with reduced gaps and Original Image with Gaps

\section{QUALITATIVE COMPARISON AND DISCUSSION}

A fingerprint image Enhancement is not aimed at producing a good visual appearance of the image but aimed at facilitating the subsequent feature detection like ridge detection and minutiae extraction and avoiding undesired side effects in the subsequent processing. Presented above the samples of enhancements to provide a visual feedback for a qualitative comparison between the original method and the enhanced images enhanced versions as delivered by the mentioned techniques. Qualitatively following parameters are used for performance analysis of the techniques discussed above.

a) PSNR: Peak Signal-to-noise ratio

The PSNR computes the peak signal-to-noise ratio and represents a measure of the peak error in decibels, between two images. This ratio is often used as a quality measurement between the original and a compressed or reconstructed image. PSNR is expressed as :

$$
P S N R=10 \log _{10}\left(\frac{R^{2}}{M S E}\right)
$$

MSE in denominator represents which represents the cumulative squared error between the reconstructed and the original image. The lower value of MSE represents the lower error in the reconstruction of the image.The mean-squared error is expressed as:

$$
: \frac{\left(\frac{\text { Original Image-Enhanced Image }}{\text { Enhanced Image }}\right)^{2}}{M+N},
$$

where M,N represents the rows and columns of Image.

b)EER (Equal Error rate): It refers to robustness and stability of algorithm This is the common value at which false acceptance rate values and false rejection rate values are equal. The False Acceptance Rate (FAR) is the success probability for an unauthorized user or a user that does not exist within a biometric system to be falsely recognized as the legally registered user. A low tolerance threshold for the biometric data to be matched leads to a lower FAR value, but to higher values of the False Rejection Rate (FRR). In contrast, the False Rejection Rate (FRR) rate is the probability of the legally registered user to be falsely rejected by the biometric system when presenting his biometric feature. High tolerance limits for the biometric data to match lead to a very low FRR value, but to higher values for the False Acceptance Rate (FAR). Both values FAR and FRR are negatively correlated. However, these measures can vary significantly depending on how one adjusts the sensitivity of the mechanism that matches the biometric. The lower the equal error rate is, the higher the accuracy is.

c)Computation Time : This metric is used to estimate the processing time involved in enhancing the image. This is observed by using cputime command of Matlab.

Following are the observations derived experimentally by applying various above enhancement techniques on public database(NIST4) on Matlab 7.8.The sample set contained 50 fingerprint images of good quality and medium quality. 
Table1. Experimental Values for Various Enhancement Techniques

\begin{tabular}{|l|l|l|l|}
\hline $\begin{array}{l}\text { Enhancement } \\
\text { Technique }\end{array}$ & EER & $\begin{array}{l}\text { Peak Signal } \\
\text { to Noise } \\
\text { ratio }\end{array}$ & $\begin{array}{l}\text { Computation } \\
\text { Time }\end{array}$ \\
\hline $\begin{array}{l}\text { Histogram } \\
\text { Equalization }\end{array}$ & $11.2 \%$ & $31.036 \mathrm{db}$ & $0.253 \mathrm{secs}$ \\
\hline WFT based & $10.978 \%$ & $35.67 \mathrm{db}$ & $0.78 \mathrm{secs}$ \\
\hline Gabor Filters & $9.8 \%$ & $43.32 \mathrm{db}$ & $0.928 \mathrm{secs}$ \\
\hline Log-Gabor & $9.767 \%$ & $36.461 \mathrm{db}$ & $0.849 \mathrm{secs}$ \\
\hline $\begin{array}{l}\text { Fast Gabor } \\
\text { Filters based }\end{array}$ & $9.89 \%$ & $38.24 \mathrm{db}$ & $0.534 \mathrm{secs}$ \\
\hline $\begin{array}{l}\text { Gabor filter in } \\
\text { Wavelet Domain }\end{array}$ & $9.976 \%$ & $39.45 \mathrm{db}$ & $0.707 \mathrm{secs}$ \\
\hline $\begin{array}{l}\text { Composite } \\
\text { Method }\end{array}$ & $9.345 \%$ & $41.56 \mathrm{db}$ & $0.894 \mathrm{secs}$ \\
\hline
\end{tabular}

Table 2.Visual Comparison of PSNR of various techniques

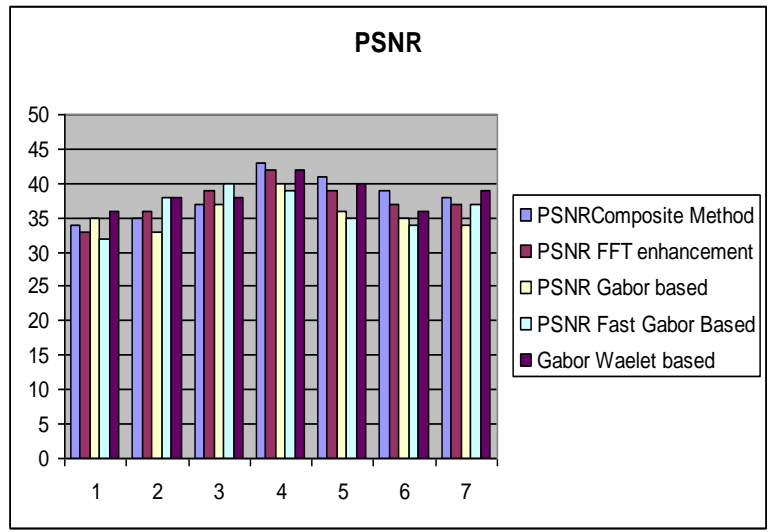

\section{CONCLUSIONS AND FURTHER SCOPE}

Various enhancement approaches have shown to improve the recognition performance in different studies. It is observed that composite method of spatial domain based and Wavelet based enhancement is giving better results. The fact is supported from the above qualitative observations given above, supported experimentally by using publicly available databases and standard minutiae matching techniques. It can be observed that there is tradeoff between the CPU times and Peak signal to noise ratio .Higher values of PSNR is expected for good recognition rate which also reduces the equal error rate .In future we will try to study and enhance the fingerprints with poor quality and heavy noise.

\section{REFRENCES}

[1] D. Maltoni, D. Maio, A. K. Jain, and S. Prabhakar (2003), Handbook of Fingerprint Recognition, Springer, New York, NY.

[2] L. Hong, Y. Wan, and A. Jain.(1998) "Fingerprint image enhancement: Algorithm and performance evaluation," IEEE Transactions on Pattern Analysis and Machine Intelligence, 20(8):777-789.

[3] Rafael C. Gonzales and Richard E. Woods, "Digital Image Processing", 3rd edition, Prentice Hall, 2009.

[4] B. G. Sherlock, D. M. Monro, and K. Millard(1994), "Fingerprint enhancement by directional Fourier Filtering," IEEE Proceedings in Visual Image Signal Processing, 141(2):87- 94.

[5] S. Chikkerur and V. Govindaraju (2005), "Fingerprint image enhancement using STFT analysis," in Proc. Int. Workshop on Pattern Recognition for Crime Prevention, Security and Surveillance, pp. 20-29.

[6] J. Yang, L. Liu, T. Jiang, and Y. Fan (2003) , "A modified gabor filter design method for fingerprint image enhancement". Pattern Recognition Letters, 24:1805-1817.

[7] C.T. Hsieh, E. Lai, and Y.C. Wang (2003), "An effective algorithm for fingerprint image enhancement base on wavelet tranform," Pattern Recognition 36, 303-312

[8] Zhengmao Ye, Habib Mohamadian, and Yongmao $\mathrm{Ye}(2007)$, "Information Measures for Biometric Identification via 2D Discrete Wavelet Transform," Proceedings of the 3rd Annual IEEE Conference on Automation Science and Engineering Scottsdale, AZ, USA, Sept 22- 25.

[9] Anto Melvin Paul and R. Mary Lourde(2006) ,"A Study on Image Enhancement Techniques for Fingerprint Identification",Proceedings of the IEEE International Conference on Video and Signal Based Surveillance, AVSS'06.

[10] Wei-Peng, Zhang, Qing-Ren. Wang, YYTang(2002), "A wavelet-based method for fingerprint image enhancement". Proceeding of the First International Conference on Machine Learning and Cybernetics, Beijing.

[11] Miao-li WEN, Yan LIANG, Quan PAN, Hong-cai ZHANG(2005), "A Gabor filter based fingerprint enhancement algorithm in wavelet domain". Communications and Information Technology, 2005. ISCIT 2005. IEEE International Symposium on 12-14 Oct. 2005. Volume 2, On page(s): 1468- 1471.

[12] A. Farina, Z.M. Kovacs-Vajna, and A. Leone(1999), "Fingerprint minutiae extraction from skeletonized binary images," Pattern Recognition, 32(5):877-889.

[13] D. Maio, D. Maltoni(1997), "Direct Gray-Scale Minutiae Detection in Fingerprints," IEEE Trans. Pattern Anal. Machine Intell., Vol. 19, No. 1, pp. 27-40.

[14] Keming Mao,Zhiliang Zhu,Huiyan Jang(2010), A fast Fingerprint Image enhancement method ,Proceeding CSO '10 Proceedings of the 2010 Third International Joint Conference on Computational Science and Optimization Volume 01,pages:222-226 
[15] Miao-li WEN, Yan LIANG, Quan PAN, Hong-cai ZHANG(2009), A Gabor Filter Based Fingerprint Enhancement Algorithm in Wavelet Domain ,(IJCSIS) International Journal of Computer Science and Information Security, Vol. 6, No. 2.

[16] K. V. Kale R. R. Manza V. T. Humbe, Fingerprint Image Enhancement using Composite Method
[17] Wei Wang , Jianwei Li, Feifei Huang, Hailiang Feng(2008), Design and implementation of Log-Gabor filter in fingerprint image enhancement, Pattern Recognition Letters $29,301-308$ 